\title{
Image Reconstruction Based on Support Similarity between Lines CoSaMP Algorithm
}

\author{
Xiuli $\mathrm{DU}^{1, \mathrm{a}}$, Binbin $\mathrm{Gu}^{1, \mathrm{~b}}$, Bo Chen ${ }^{1, \mathrm{c}}$,Xing $\mathrm{Hu}^{1, \mathrm{~d}}$ \\ ${ }^{1}$ Key Laboratory of Communication Networks and Information Processing, Dalian University, Dalian, 116622, \\ China

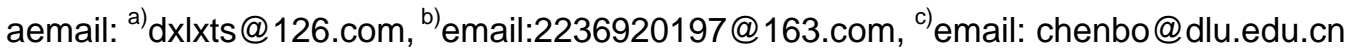

Keywords: Image reconstruction, Support similarity, Lines CoSaMP algorithm

\begin{abstract}
The algorithm of compressive sampling matching pursuit (CoSaMP) is the performance of this algorithm is restricted to the choose of it's initial support set. If inaccurately choose the initial support set, it will not only affect the accuracy of reconsitution, but also reduce the speed of reconsitution. In order to solve this problem, the stucture of image in sparse domain is introduced into CoSaMP algorithm, and the concept of support set similarity is presented in this paper, and we raised one kind of CoSaMP algorithm which is based on Support Similarity between Lines and uses the high similarity between the adjoining rows in one digital image. The result of this experiment shows that under the condition with same sampling frequency, when don't increase the time complexity of the algorithm, the algorithm which we have raised has higher quality in reconsitution and the peak signal to noise ratio enhances $0.6 \sim 2.5 \mathrm{~dB}$, compared with the traditional CoSaMP algorithm.
\end{abstract}

\section{INTRODUCTION}

With the rapid development of modern sensor technology, the size of the image from the original $\mathrm{K}$ bytes increased to the $\mathrm{M}$ bytes, or even the $\mathrm{G}$ byte. Huge data volume to the image storage and transmission caused great inconvenience, image compression technology is particularly important. However, the traditional image compression technology is compressed after sampling, which will result in a large degree of waste of resources. Therefore, how to solve the real-time image compression problem is an urgent need to solve the problem, and by Donoho and Candes proposed compressive sensing theory[1-3] to solve this problem provides a good theoretical basis.

At present, the existing reconstruction algorithms mainly include convex optimization algorithm [4], greedy iterative algorithm [5-6] and combinatorial algorithm [7]. The CoSaMP [8-11] algorithm has a good theoretical basis and strong anti-noise ability, but in the signal reconstruction, the reconstruction performance is affected by the initial support set selection, the initial support set selection is not accurate not only affect the reconstruction accuracy but also Reduce the reconstruction speed. In [12], an improved CoSaMP algorithm is proposed to further optimize the support set by the Dice coefficient selection criterion and improve the quality of reconstruction. we propose an image reconstruction algorithm with sparse support set in [13], On the basis of known image reconstruction, the initial estimation of the current image is obtained, which improves the accuracy of the selection of the image sequence support set, but requires the image sequence to be gradual and has some limitations. In [14], an improved sampling and matching algorithm based on pseudo inverse processing is proposed, Before the restoration, through the pseudo-processing of the observation matrix, to enhance the non-correlation between atoms, thereby improving the quality of reconstruction. The above algorithm can improve the quality of reconstruction, but increase the time complexity of the algorithm.

In this paper, based on the similarity between adjacent rows of digital images without increasing the complexity of the algorithm, the initial support set selection problem of the compression sampling matching algorithm is used to optimize the selection of the initial support set. Propose an algorithm based on Support Similarity between Lines Compressive Sampling Matching Pursuit. 


\section{COSAMP ALGORITHM}

The idea of a greedy iterative algorithm is to gradually approximate the estimated signal based on a greedy iteration criterion. OMP is proposed earlier for the greedy iteration algorithm in compression perception, Each iteration, the residual and the perception of the matrix of the largest index value corresponding to the atoms were selected to support the concentration, the disadvantage is that once the atoms are added to the support set can not be changed. Then, the CoSaMP algorithm is proposed by Needell et al., Which introduces the "backtracking" thought into the updating of the atomic support set. First, by calculating the correlation between the signal residual and the inductance matrix, and selecting the $2 \mathrm{~K}$ atoms To expand the support set, And then according to the size of the recovery factor to eliminate some of the atoms, but must ensure that the final support of the remaining number of atoms and the signal sparse degree of $\mathrm{K}$ equal size.The concrete implementation steps of the CoSaMP algorithm are as follows

\section{SSLCOSAMP IMAGE RECONSTRUCTION ALGORITHM}

\section{Image sparse domain correlation analysis}

It has been shown that the correlation between adjacent pixels in the digital image is strong, and the correlation coefficient between the two adjacent pixels or adjacent two rows is 0.9 or more [15]. If the image has a strong correlation on the sparse domain, the selection of the initial support set can be realized by using the correlation on the sparse domain. In order to analyze the correlation of the image in the sparse domain, the Lena image with the energy of each band is selected as the test image, and the sparse coefficient is obtained by the transformation of the orthogonal matrix (DCT transform matrix). In order to better express the coefficient distribution law through the image, the coefficients are logarithmically transformed, and the coefficient distribution after transformation is shown in Fig.1 It can be seen from the figure that the coefficient distribution has a certain regularity. In order to further verify the distribution of the support set, the sparse coefficients are binarized by polynomial (5) ( $\varepsilon$ is the sparse threshold, the value is $\varepsilon=13$ ). The resulting results are shown in Fig 2.

$$
\theta(i, j)= \begin{cases}255, & |\theta(i, j)| \geq \varepsilon \\ 0, & |\theta(i, j)|<\varepsilon\end{cases}
$$

In Fig. 2 the white area corresponds to a value of 255 and the black area corresponds to a value of zero. And white areas are mostly distributed in the left area of the image, that is, non-zero elements in the left area is more intensive. This graph also shows the distribution of the support set under ideal conditions. The above shows that the distribution of nonzero elements in the sparse domain of the actual image has some regularity, and the adjacent rows have great similarity in the sparse domain.

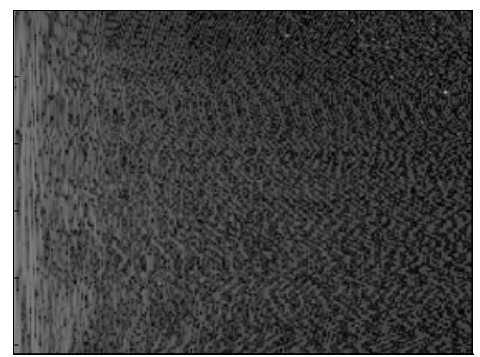

FIGURE 1. Lena image sparse domain coefficient distribution

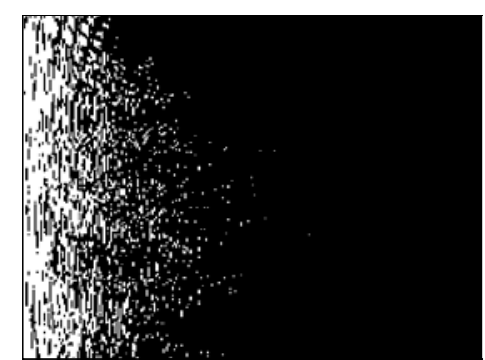

FIGURE 2. Lena image sparse domain coefficient binarization distribution

In order to clarify the similarity of adjacent lines, it will be quantitatively analyzed. First, through the covariance matrix to obtain the correlation coefficient of two adjacent lines, the results shown in Fig.3. It can be seen from Fig 3, the inter-row correlation coefficient is above 0.9, are highly correlated.

Use (6) to show the similarity of the adjacent row support set:

$$
\operatorname{Sim}(i, i+1)=\frac{\sum \theta(i,:) \cap \theta(i+1,:)}{K}
$$


In the formula (6), the number of elements in the intersection of two adjacent nonzero coefficients in the sparse domain of the image is denoted by the sparse degree $\mathrm{K}$ so that the value range $[0,1]$ of the equation. Since the index position of the sparse coefficient corresponds to the position of the corresponding support set vector, the formula can describe the similarity of the support set of two rows adjacent to the image. Fig.4 shows the similarity curve for the adjacent lines of the Lena image. It can be obtained from Fig. 4 that the similarity of adjacent row support set in sparse domain is above 0.7. Therefore, the support set that can be used on the previous row can be used as a prerequisite information for the current row reconstruction.

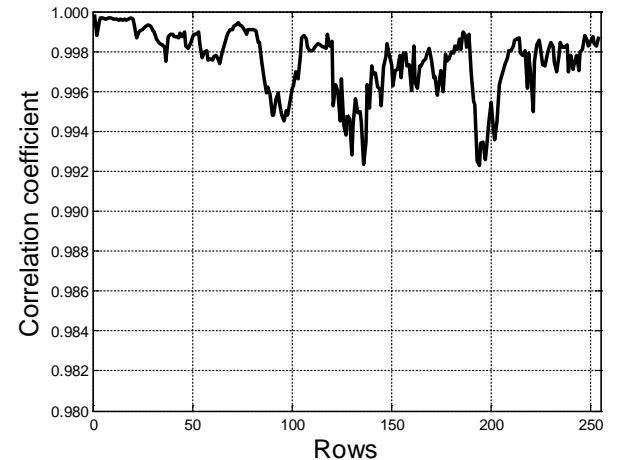

FIGURE 3. Lena image sparse domain adjacent row correlation coefficient curve

In order to verify the universality of this conclusion, this paper makes a mathematical statistics on the correlation coefficient and support set similarity of a large number of people, buildings and landscape images, and the results are shown in Fig. 5 and Fig. 6. Through the histogram we can see that such images have strong correlation, and can be reconstructed by the reconstruction algorithm proposed in this paper.

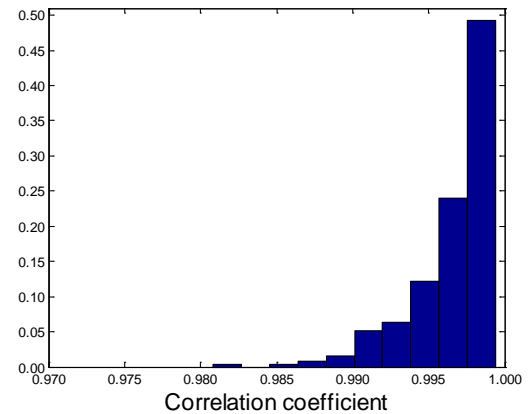

FIGURE 5. Image Correlation Coefficient Probability Statistical Histogram

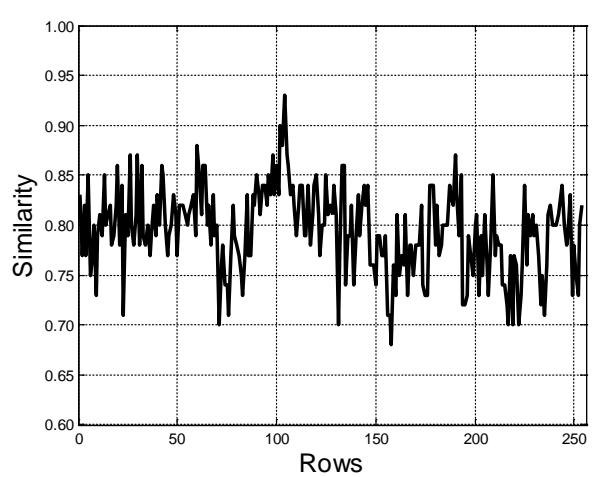

FIGURE 4. Lena image sparse domain adjacent row support set similarity curve 
(1) Calculating the residual product $r^{(t-1)}$ and the inner product $g^{(t)}=A^{T} r^{(t-1)}$ of the sensing matrix A ;

(2) Find the index value $T_{2 k}=\underset{i=\{1,2, \ldots, N\}\}}{\arg \max }\left|g^{(t)}[i]\right|$ corresponding to the $2 \mathrm{~K}$ element with the largest absolute value in the inner product $g^{(t)}$, update the set of index values $\Lambda^{(t)}=\hat{\Lambda}^{(t-1)} \cup\left\{T_{2 K}\right\}$ and the corresponding set of atoms $A_{\Lambda^{(t)}}=A_{\hat{\Lambda}^{(t-1)}} \cup\left\{a_{T_{2 K}}\right\}$;

(3) The pseudo inverse matrix $A_{\Lambda^{(t)}}^{\dagger}=\left(A_{\Lambda^{(t)}}^{T} A_{\Lambda^{(t)}}\right)^{-1} A_{\Lambda^{(t)}}^{T}$ of the selected atomic set $A_{\Lambda^{(t)}}$ is obtained, Thus using the least squares method to obtain $\theta^{(t)}=A_{\Lambda^{(t)}}^{\dagger} y=\left(A_{\Lambda^{(t)}}^{T} A_{\Lambda^{(t)}}\right)^{-1} A_{\Lambda^{(t)}}^{T} y$;

(4) Picking the $\mathrm{K}$ elements with the largest absolute value in the $\theta^{(t)}$ element to form the index set $\hat{\Lambda}^{(t)}$ and its corresponding set of atoms $A_{\hat{\Lambda}^{(t)}}$;

(5) The sparse approximation coefficient $\hat{\theta}^{(t)}=\left(A_{\hat{\Lambda}^{(t)}}^{T} A_{\hat{\Lambda}^{(t)}}\right)^{-1} A_{\hat{\Lambda}^{(t)}}^{T} y$ is obtained by the least squares method, and the residual $r^{(t)}=y-A \hat{\theta}^{(t)}$ is updated;

(6) It is judged whether or not the iteration stop condition $t<K$ is satisfied, the reconstructed signal $\hat{x}=\psi \hat{\theta}^{(t)}$ is calculated if the condition is satisfied, Otherwise, make the iteration number $\mathrm{x}$, and jump to (1).

\section{EXPERIMENTS}

In order to verify the performance of the proposed algorithm to reconstruct the image, this chapter uses the different types of images with the size of $256 \times 256$ in the Matlab simulation environment: Lena images with equal energy bands (Fig.7) and Boat images with high frequency energy (Fig. 8) are used as test images. The CoSaMP algorithm and the SSLCoSaMP algorithm proposed in this paper are used to reconstruct the test image under different sampling rate. The sampling rate $\mathrm{M} / \mathrm{N}$ is set to $0.1,0.2,0.3,0.4$ and 0.5 for simulation experiments. The performance of the SSLCoSaMP algorithm is verified by comparing the quality of the image reconstruction with the run time. In the experiment, the DCT transform matrix is used to sparse transform the test image, and the Gaussian random matrix is selected as the measurement matrix to compress the samples. Due to the randomness of the measurement matrix, the experiment is run 200 times in each experiment. The average of the results.

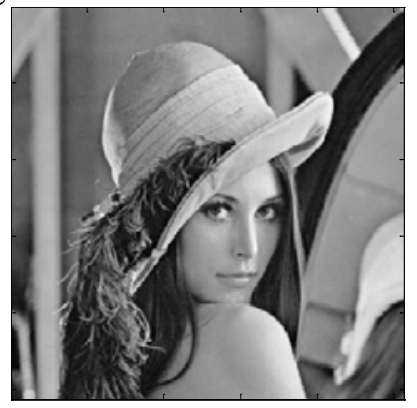

FIGURE 7. Lena

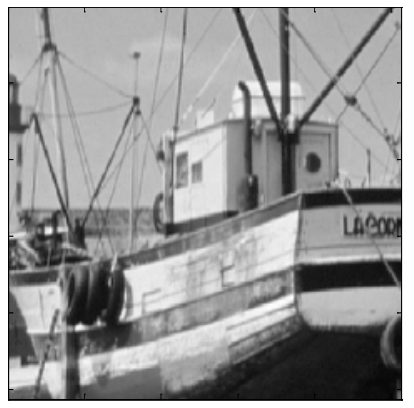

FIGURE 8. Boat

\section{Comparison of Reconstructed Images}

CoSaMP algorithm and the proposed SSLCoSaMP algorithm reconstruction effect shown in Fig.9 to Fig.12, the sampling rate $(\mathrm{M} / \mathrm{N})$ is 0.3 , CoSaMP algorithm reconstruction effect is poor. Some of the blurring and noise in the reconstructed image results in the details of the image that can not be reproduced, such as the feather lines on the Lena hat and the thin rods of the Boat distance. Compared with CoSaMP algorithm, SSLCoSaMP algorithm introduces less shadow and better preserves the detail information in the image. With the increase of the sampling rate, the effect of the two groups is getting closer and closer, and when the sampling rate $(\mathrm{M} / \mathrm{N})$ is 0.4 and 0.5 , it is impossible to distinguish the slight difference from the visual. In order to better quantify the effect of image reconstruction. In this paper, the peak signal to noise ratio (PSNR) is used as the evaluation index, as shown in Table 1. 


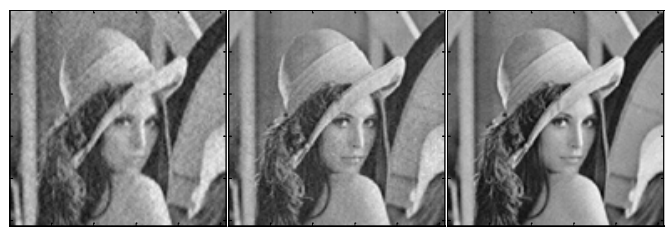

(a) $\mathrm{M} / \mathrm{N}=0.3$

(c) $\mathrm{M} / \mathrm{N}=0.5$

FIGURE 9. Reconstruction of Lena Image of CoSaMP Algorithm

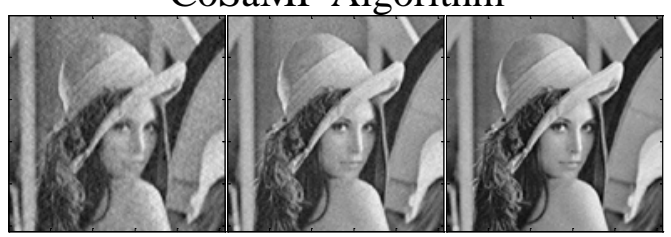

(a) $\mathrm{M} / \mathrm{N}=0.3$

(b) $\mathrm{M} / \mathrm{N}=0.4$

(c) $\mathrm{M} / \mathrm{N}=0.5$

FIGURE 10. Reconstruction of Lena Image of SSLCoSaMP Algorithm

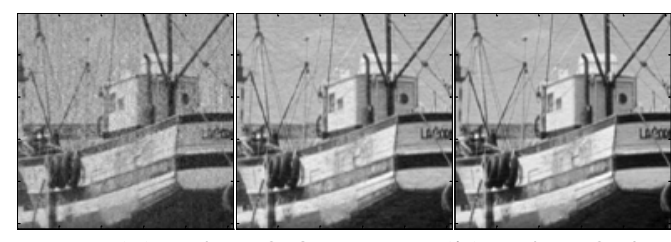

(a) $\mathrm{M} / \mathrm{N}=0.3$

(b) $\mathrm{M} / \mathrm{N}=0.4$

(c) $\mathrm{M} / \mathrm{N}=0.5$

FIGURE 11. Reconstruction of Boat Image of CoSaMP Algorithm

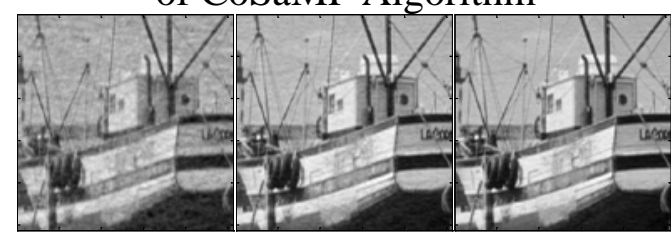

(a) $\mathrm{M} / \mathrm{N}=0.3$

(b) $\mathrm{M} / \mathrm{N}=0.4$

(c) $\mathrm{M} / \mathrm{N}=0.5$

FIGURE 12. Reconstruction of Boat Image of SSLCoSaMP Algorithm

TABLE 1. Comparison of PSNR after Reconstruction of CoSaMP Algorithm and SSLCoSaMP Algorithm $(\mathrm{dB})$

\begin{tabular}{ccccccc}
\hline & $\begin{array}{c}\text { Sampling } \\
\text { Rate }\end{array}$ & 0.1 & 0.2 & 0.3 & 0.4 & 0.5 \\
\hline \multirow{2}{*}{ Lena } & CoSaMP & 18.48 & 23.13 & 26.46 & 28.46 & 31.48 \\
& SSLCoSaMP & 20.53 & 24.85 & 27.50 & 29.50 & 32.21 \\
\hline \multirow{2}{*}{ Boat } & CoSaMP & 14.35 & 21.26 & 26.02 & 32.21 & 34.59 \\
& SSLCoSaMP & 16.82 & 22.40 & 26.97 & 32.98 & 35.22 \\
\hline
\end{tabular}

PSNR is an important indicator of the similarity of two images. The larger the value is, the higher the similarity is. When the sampling rate is low, the SSLCoSaMP algorithm proposed in this paper is about $2 \mathrm{~dB}$ higher than that of the CoSaMP algorithm. With the increase of the sampling rate, the reconstruction effect of the two algorithms is improved, When the sampling rate $(\mathrm{M} / \mathrm{N})$ is 0.5, the SSLCoSaMP algorithm is also nearly 0.5dB higher than the CoSaMP algorithm. This result also corresponds to the reconstructed image at different sampling rates. When the sampling rate is low, the number of compressed samples is small, too much useful information has been lost at the time of sampling, and the number of samples has not reached the requirement of accurate image restoration, which can not achieve the desired effect. With the increase of the sampling rate, more useful information is preserved in the sampling, the reconstruction effect is also ideal. CoSaMP algorithm in the atomic support set selection, the introduction of too many false estimates of atoms, reconstruction results are poor. The SSLCoSaMP algorithm uses the uplink a priori support set as the initial condition of the current row of atomic support set, the support set is more accurate, the reconstruction effect is good.

\section{Time comparison of reconstructed images}

The length of the algorithm is also an important measure of the performance of the reconstruction. Fig.13 and Fig.14 show the CoSaMP algorithm and the run time of the proposed SSLCoSaMP algorithm at different sampling rates. 


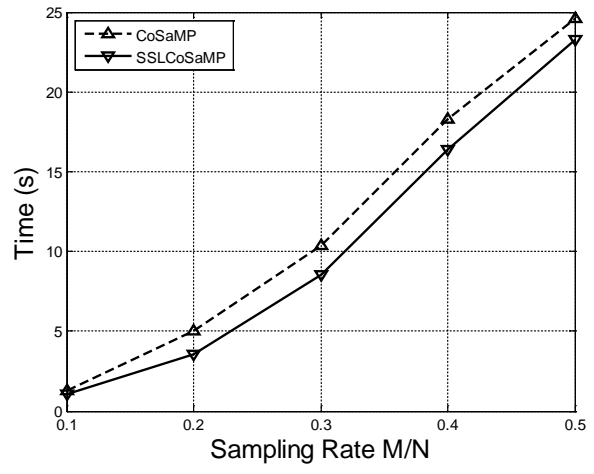

FIGURE 13. Lena Image run time comparison

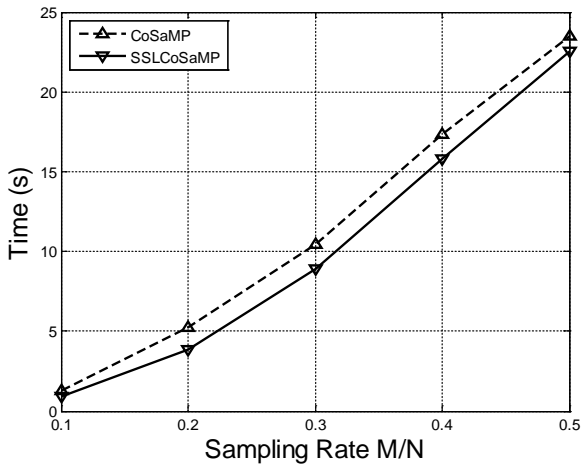

FIGURE 14. Boat Image run time comparison

From the Fig, the SSLCoSaMP algorithm runs better than the CoSaMP algorithm. This is because the SSLCoSaMP algorithm preserves the upstream reconstructed atomic support set to the current row, which is used to initialize the atomic support set of the current reconstructed row to find the best support set more quickly.

\section{CONCLUSION}

In this paper, a compression sampling matching algorithm based on sparse support set is proposed to solve the problem of performance instability caused by the initial support set of CoSaMP reconstruction algorithm. The concept of similarity degree of support set is established, and it is proposed to reconstruct the image by using the support set of the previous row in the image as the prior information reconstructed. The experimental results of different images show that under the same conditions, the algorithm improves the time complexity of CoSaMP algorithm by $0.6 \sim$ $2.5 \mathrm{~dB}$. However, the algorithm reconstructed images require strong inter-row correlation, otherwise the reconstruction performance will be constrained. How to use the reconstruction factor spatial gradation to further improve the precision of reconstruction will be the focus of the next step.

\section{ACKNOWLEDGMENTS}

Communication author : Binbin Gu; Telephone number : 15842600760; E-mail : 2236920197@qq.com .

\section{REFERENCES}

[1] J. E Cand $\cdot \mathrm{s}$. Compressive sampling[A]. Proceedings of the International Congress of Mathematicians[C]. Madrid, Spain, 2006,3:1433-1452.

[2] D L Donoho. Compressed sensing[J]. IEEE Trans. On Information Theory, 2006,52(4):12891306.

[3] D L Donoho, Y Tsaig. Extensions of compressed sensing[J]. Signal Processing, 2006,86(3):533548.

[4] Mingli Jing, Xueqin Zhou, Chun Qi. Quasi-Newton Iterative Projection Algorithm for Sparse Recovery[J]. Neurocomputing, 2014,11,144(20): 169-173.

[5] Hong Fang,Hairong Yang. Greedy algorithm and compression perception theory. Journal of Automation, 2011,12 (37): 1413-1421.

[6] J. Wang, S. Kwon, and B. Shim. Generalized orthogonal matching pursuit. to appear in IEEE Trans. Signal Process. Dec. 2012.

[7] Bijit Kumar Das and Mrityunjoy Chakraborty. Sparse Adaptive Filtering by an Adaptive Convex Combination of the LMS and the ZA-LMS Algorithms. IEEE Trans. on Circuits and Systems - I: Regular Papers, 2014,5, 61(5): 1499-1507.

[8] D Needell, J A Tropp. CoSaMP: Iterative signal recovery from incomplete and inaccurate samples[J]. Appl and CompHarm. Anal,2009,26(3):301-321. 
[9] Xiaowei Zhang, Ming Li. Based on SWCoSaMP algorithm for sparse signal reconstruction [J]. Signal Processing, 2012,6,28 (6): 886-893.

[10] Mark A. Davenport, Deanna Needell, Michael B. Wakin. Signal Space CoSaMP for Sparse Recovery With Redundant Dictionaries[J]. IEEE Transactions on Information Theory,2013,59(10): 6820 - 6829 .

[11] Raja Giryes, Michael Elad. RIP-Based Near-Oracle Performance Guarantees for SP,CoSaMP, and IHT[J]. IEEE Transactions on Signal Processing,2012,60(3): 1465 - 1468

[12] Danya Liang, Hongwei Li, QI Li etc. An Improved Compression-aware Reconstruction Algorithm [J]

[13] Xingxiu Li. Journal of Nanjing University of Science and Technology (Social Science Edition) Journal of Nanjing University of Science and Technology (Natural Science Edition) Journal of Nanjing University of Science and Technology (Natural Science Edition) 2012,12,36(6):973-978.

[14] Xinyan Hui,Yun Lin. Improved Compression Sampling Matching Tracking Algorithm [J]. Journal of Computer Applications, 2015,35 (S1): 331-334.

[15] Xuelong Hu. Digital Image Processing (Third Edition) [M]. Electronic Industry Press,2014,8. 